\title{
Therapeutic effects of micheliolide on a murine model of rheumatoid arthritis
}

\author{
HUA XU, JIAN WANG, CHIJUAN WANG, GUOQIANG CHANG, YANI LIN, HONGJU ZHANG, \\ HAIRUI ZHANG, QINGHUA LI and TIANXIANG PANG
}

\begin{abstract}
State Key Laboratory of Experimental Hematology, Institute of Hematology and Blood Diseases Hospital, Chinese Academy of Medical Sciences and Peking Union Medical College, Tianjin 300020, P.R. China
\end{abstract}

Received February 8, 2014; Accepted September 29, 2014

DOI: $10.3892 / \mathrm{mmr} .2014 .2767$

\begin{abstract}
Rheumatoid arthritis (RA) is a systemic autoimmune disease and collagen-induced arthritis (CIA) is an animal model for RA. Micheliolide (MCL) is a novel compound with strong anti-inflammatory effects. The present study was conducted to evaluate the therapeutic effects of MCL on RA. Mice were randomly divided into four groups and the CIA model mice were treated with methotrexate (MTX), MCL and dimethyl sulfoxide. A score associated with the severity of arthritis was assigned on alternate days from the 22nd day for 60 days. Histopathological changes and the serum levels of cytokines were measured on day 85 . The results demonstrated that the MCL treatment group had arthritis scores lower than the CIA group and higher than the MTX group; compared with the CIA group, MCL and MTX significantly reduced the swelling of the paws and suppressed the degeneration of articular cartilage. Expression levels of macrophage colony-stimulating factor (M-CSF), tissue inhibitors of metalloproteinases-1 (TIMP-1) and complement component 5a (C5/C5a) were lower in the mice with arthritis compared with normal mice, however, following treatment with MCL and MTX, all the mice exhibited significant recovery to differing degrees. Unlike the MTX group, the MCL group failed to recover the level of soluble intercellular adhesion molecule-1. In addition, the cytokine of B-lymphocyte chemoattractant (BLC) solely presented in the MCL group. These results suggest that MCL may be considered for use as a novel therapeutic treatment against RA and that changes in the expression of cytokines C5/C5a, TIMP-1, M-CSF and BLC
\end{abstract}

Correspondence to: Professor Tianxiang Pang, State Key Laboratory of Experimental Hematology, Institute of Hematology and Blood Diseases Hospital, Chinese Academy of Medical Sciences and Peking Union Medical College, 288 Nanjing Road, Tianjin 300020, P.R. China

E-mail: pang@ihcams.ac.cn

Key words: micheliolide, rheumatoid arthritis, methotrexate, collagen-induced arthritis may underlie the mechanism by which MCL effects changes in this disease.

\section{Introduction}

Rheumatoid arthritis (RA) is a chronic autoimmune disease affecting approximately $1 \%$ of the population world-wide, in a female:male ratio of $2.5 / 1$. Over 60 million people suffer from this disorder, which is characterized by arthrosynovitis and erosive destruction of peripheral joints. These clinical manifestations are chronic, occur symmetrically and synovitis is present in multiple joints (1-4). To date, the cause of RA has not been fully elucidated. Although a number of drugs are used for the clinical treatment of RA, an effective drug with few side effects and of low cost is still lacking. Micheliolide (MCL) is a new effective compound for the treatment of RA; it is a guaianolide sesquiterpene lactone (5) that can be isolated from magnoliaceae (6) and semi-synthesized from parthenolide with $90 \%$ yield (7). Parthenolide is a traditional drug, which originated in Europe and has demonstrated potent anticancer and anti-inflammatory activities (8). Compared with parthenolide, MCL exhibits greater plasma stability with a more sustained release and superior in vivo efficacy (9) has a low toxicity, low cost and strong anti-inflammatory effects. Methotrexate (MTX), is an almost unsurpassed drug for the clinical treatment of RA and it has been supported for its safety and convenience, since it is amenable to administration by the oral route (10-14). The collagen-induced arthritis (CIA) mouse model is the most commonly studied autoimmune model of RA. It is induced by immunization with an emulsion of complete Freund's anduvant and type II collagen (CII) (15). In the present study, the effects of MCL and MTX on type II collagen-induced arthritis (CIA) in mice were investigated as well as evaluating whether MCL may be used as a new drug for the treatment of RA.

\section{Materials and methods}

Animals. Experiments were performed according to the Declaration of Helsinki guidelines and were approved by the institutional biomedical research ethics committee in Laboratory Animal Center of Institute of Hematology and Blood Diseases Hospital, Chinese Academy of Medical Sciences (Tianjin, China). A total of 40 specific-pathogen free male DBA/1 mice, 
aged 6-7 weeks, were purchased from Beijing HFK Bioscience Co., Ltd (Beijing, China).

CIA and treatment. In total, forty DBA/1 mice were randomly divided into four groups and numbered. The groups were termed normal (Nor), model (CIA), MTX and MCL groups. On the first day, DBA/1 mice (with the exception of the normal group mice) were immunized by intradermal injections at the base of the tail with an emulsion containing $100 \mu \mathrm{g}$ of immunization grade bovine type II collagen (CII; Chondrex Inc., Richmond,WA,USA) in complete Freund's adjuvant containing heat-killed mycobacterium butyricum (Chondrex Inc., Richmond, WA, USA). On day 21 , a booster containing $100 \mu \mathrm{g}$ bovine $\mathrm{CII}$ in incomplete Freund's adjuvant (Chondrex Inc.) was administered (15). Drugs were administered intraperitoneally (IP) every two days from day 22. The normal group did not receive treatment; the model group was administered with equal volumes of solvent, DMSO (Tianjin Fengchuan Chemical Reagent Science and Technology Co.,Ltd., Tianjin, China); the MTX group was treated with $6.6 \mathrm{mg} / \mathrm{kg}$ MTX (Shanxi Powerdone Pharmaceutics Co., Ltd, Beijing, China) solution (dissolved in saline) (16); the MCL group was treated with $30 \mathrm{mg} / \mathrm{kg}$ MCL (provided by Prof. Chen Yue, College of Pharmacy, Nankai University, Tianjin, China) solution (dissolved in DMSO). All treatments were administrated 28 times to all mice in the corresponding group.

Evaluation of the disease. To evaluate the disease, the mice were measured on alternate days for 60 days following the booster immunization. The clinical severity of paw inflammation was scored periodically by the arthritis scores. The arthritis scores were evaluated as follows: 0 , Normal; 1 , swelling and redness of the paw or one digit; 2 , two joints involved; 3 , more than two joints involved and 4, severe arthritis of the entire paw and digits. The final score for each mouse was the sum of the four paws (17).

Histopathology. The knees and paws of the mice were severed, fixed in phosphate-buffered saline containing $4 \%$ formaldehyde, decalcified in $10 \%$ ethylenediaminetetraacetic acid and embedded in paraffin. The tissues were sliced and stained with hematoxylin and eosin ( $\mathrm{H}$ and $\mathrm{E})$. Histopathological changes were observed under a light microscope (Eclipse TE300, Nikon Corporation, Tokyo, Japan)' and scored by two independent observers using the following scoring systems: Inflammation was scored on a scale of 0-2 as follows: 0 , Normal, 1 , local inflammatory infiltration and 2, marked infiltration with lymphoid aggregates and edema. Pannus formation and articular cartilage damage were scored on a scale of 0-2 as follows: 0, Normal, 1 , synovial proliferation adjacent to cartilage but no articular cartilage damage and 2, synovial proliferation and articular cartilage damage (18).

Measurement of serum cytokines. The cytokines in the mouse serum were measured using mouse cytokine array panel A (R\&D Systems, Minneapolis, MN, USA). Briefly, blood samples were allowed to clot for $1 \mathrm{~h}$ at room temperature prior to incubation for $4 \mathrm{~h}$ at $4^{\circ} \mathrm{C}$ and subsequently centrifuged at $1,500 \mathrm{x}$ g for 10 min by (Sorvall Legend Micro 17R, Thermo Fisher Scientific, Waltham, MA, USA). Serum was removed and assayed by mouse cytokine array panel A, according to the manufacturer's

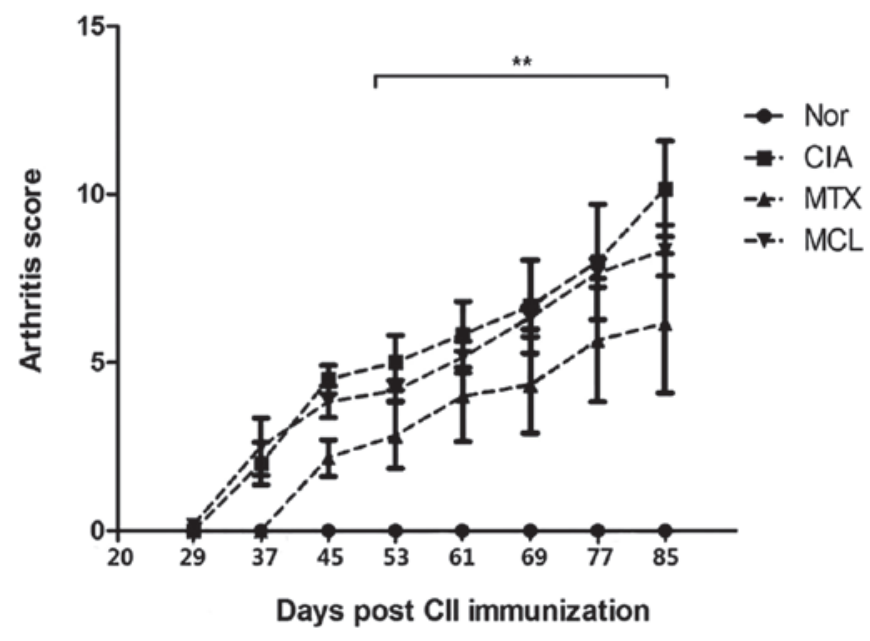

Figure 1. Arthritis scores of the CII-immunized mice and the normal mice. Error bars have been omitted for clarity of presentation. ${ }^{* *} \mathrm{P}<0.01$ compared with CIA group. This data is representative of three separate experiments. CIA, Collagen induced arthritis; CII, type II collagen; MTX, methotrexate; MCL, micheliolide; Nor, normal.

instructions. Membranes were blocked for $1 \mathrm{~h}$, samples were subsequently added to an antibody detection cocktail (R\&D Systems), mixed and incubated at room temperature for $1 \mathrm{~h}$ prior to overnight incubation at $4^{\circ} \mathrm{C}$. Samples were subsequently washed, streptavidin-horseradish peroxidase conjugate working solution was added and incubated for $30 \mathrm{~min}$ at room temperature on a rocking platform shaker. Following washing, samples were incubated for 1 min using a Chemi Reagent Mix (R\&D Systems), samples were then exposed to X-ray film for 1-10 min and the scanned results were analyzed using Adobe Photoshop CS4 (Adobe Systems, Inc., San Jose, CA, USA).

Statistical analysis. All data were summarized and are presented as the mean \pm standard deviation (SD) and analyzed using the GraphPad Prism Version 5 software program (GraphPad Prism, San Diego, CA, USA). Group comparisons were performed using the unpaired Student's $t$-test. $\mathrm{P}<0.05$ was considered to indicate a statistically significant difference.

\section{Results}

Evaluation of the disease. Compared with the model group, MCL and MTX were able to significantly reduce swelling of the paws and suppress the degeneration of articular cartilage, as demonstrated by the lower arthritis scores compared with the model group. The arthritis scores of the MCL group were slightly higher than those of the MTX group, suggesting that the therapeutic effect of MCL on CIA was less significant than for MTX (Fig. 1).

Effects of MCL on histopathological changes. $\mathrm{H}$ and $\mathrm{E}$ staining indicated that the joints in the normal group exhibited no pathological changes (Fig. 2A: i-iii). However, staining revealed multiple pathological changes in the model group, including significant inflammatory cell infiltration (Fig. 2A: iv), hyperplasia of synovial cells, formation of granulation 
A

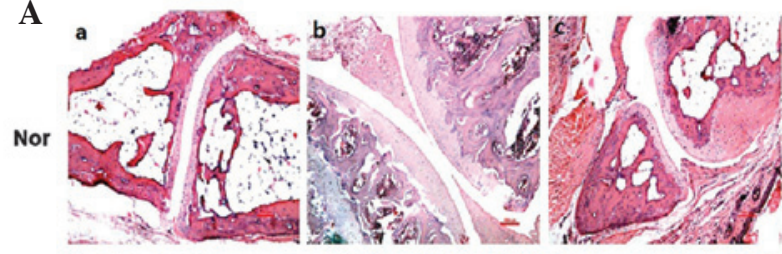

CIA

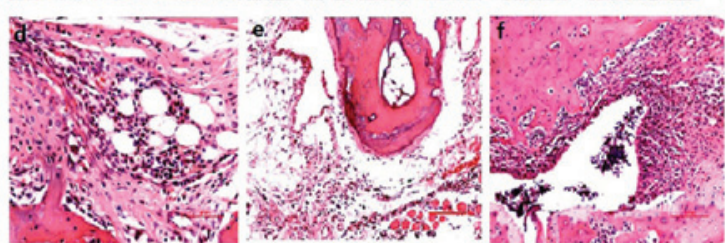

MTX
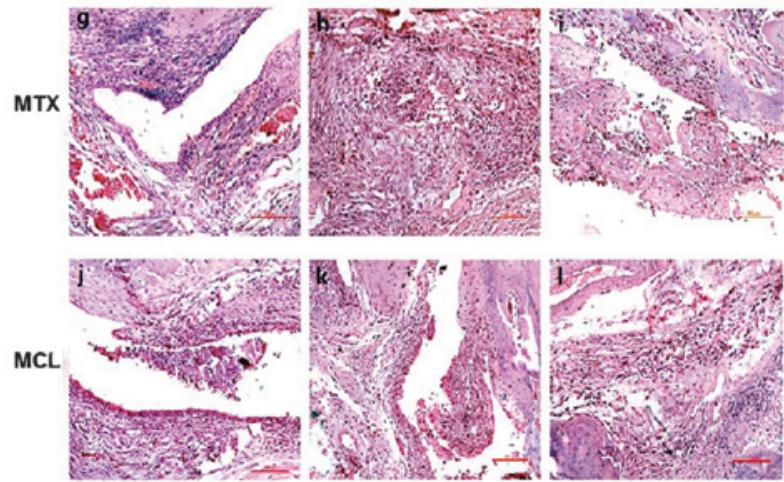

B

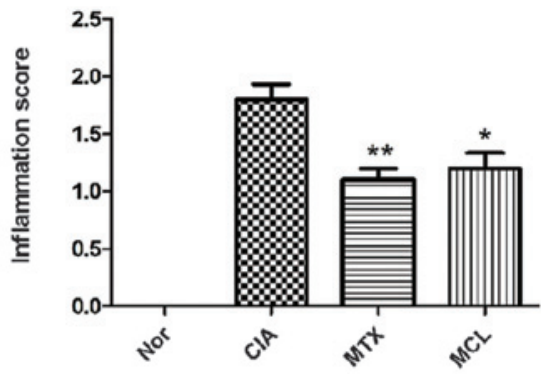

C

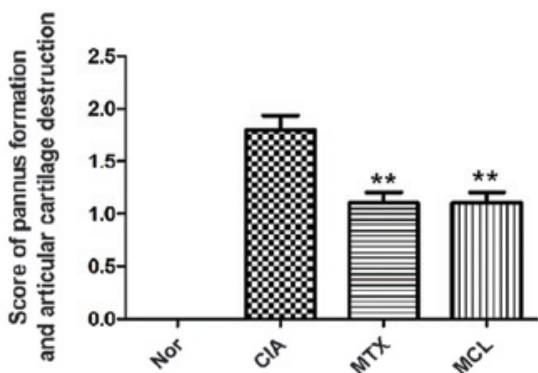

Figure 2. Joint tissues with hematoxylin and eosin staining of the four groups. (A: i-iii) Normal joint tissue present from normal control group; (A: iv-vi), (A: vii-ix) and (A: X-xii) are the corresponding specimens from CIA control group, MTX control group and MCL group, respectively. (A: iv-xii) Indicate infiltration by inflammatory cells, synovitis cell infiltration and hyperplasia of fibrous tissue. (B) Inflammation score of CIA control group, MTX control group and MCL group. Data is presented as the mean \pm SEM of clinical scores. ${ }^{*} \mathrm{P}<0.05,{ }^{* * *} \mathrm{P}<0.01$ compared with CIA group. (C) The score of pannus formation and articular cartilage destruction of CIA control group, MTX control group and MCL group. Data is presented as the mean \pm SEM of clinical scores. ${ }^{* *} \mathrm{P}<0.01$ compared with CIA group. This data is representative of three separate experiments, which yielded similar results. CIA, collagen induced arthritis; MTX, methotrexate; MCL, micheliolide; SEM, standard error of the mean.

A

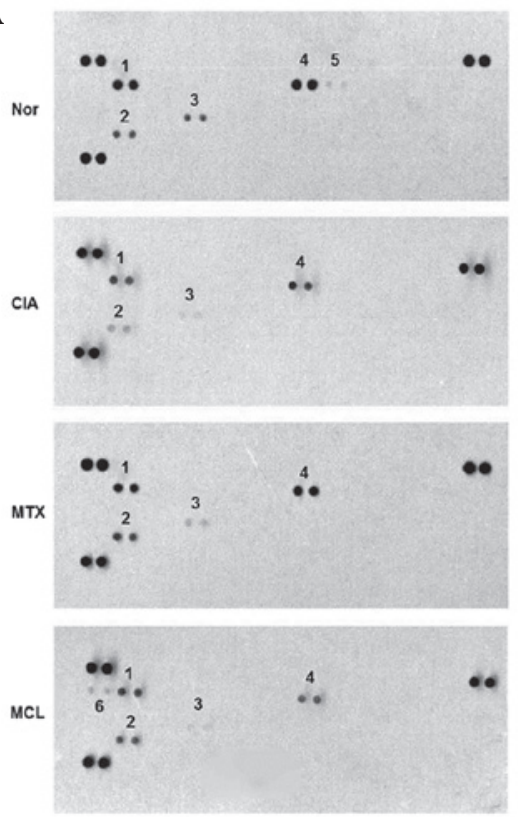

B

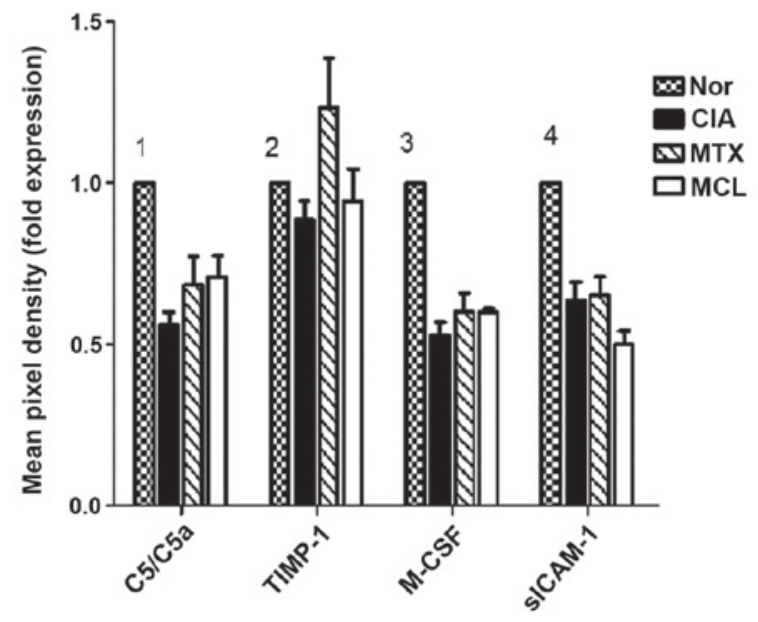

Figure 3. Mouse cytokine test for the four groups of mice. (A) Six cytokines are presented in this experiment and they are denoted 1-6. Cytokines 1-4 are present in all four groups; these are C5/C5a, TIMP-1, M-CSF and (sICAM-1); cytokine 5 refers to interferon- $\gamma$, only shown in the normal control group; cytokine 6 is B-Lymphocyte chemoattractant, only present in the MCL group. (B) The result of M-CSF is the same as that of C5/C5a: The normal control group is the highest and the CIA control group is the lowest, while the MCL group is between the CIA control group and the MTX control group. ** $\mathrm{P}<0.01$ compared with the CIA group; TIMP-1, the MCL group and the MTX group are higher than the CIA control group as well as marginally higher than the normal control group and are very similar to each other. ${ }^{* *} \mathrm{P}<0.01$ compared with the CIA group; sICAM-1; the MCL group is the lowest group. This data is representative of three separate experiments, which yielded similar results. CIA, Collagen induced arthritis; MTX, methotrexate; MCL, micheliolide; SEM, standard error of the mean; C5/C5A, complement component 5a; TIMP-1, tissue inhibitors of metalloproteinases-1; M-CSF, macrophage colony-stimulating factor; sICAM-1, soluble intercellular adhesion molecule-1. 
tissue (Fig. 2A: v) and necrotic tissue present in the articular cavity (Fig. 2A: vi). The pathological changes in the MTX and MCL group were weaker than for the model group. In the MTX group, synovial cells of fibrous tissue exhibited infiltration and hyperplasia (Fig. 2A: vii), inflammatory cells infiltrated the tissues surrounding the joints (Fig. 2A: viii) and inflammatory cells emerged from articular cavities (Fig. 2A: ix). The pathological changes in the MCL group were similar to those of the MTX group (Fig. 2A: x-xii). The inflammation score, the score of pannus formation and articular cartilage destruction further indicated this (Fig. 2B, C). The present results revealed that MCL as well as MTX could remit the pathological changes of CIA.

Effects of MCL on serum cytokines. While forty cytokines were assessed in this experiment, only six of them were detectable in serum and are presented in the results (Fig. 3A). These are denoted 1-6. Cytokines 1-4 were all present in the four experimental groups, these were complement component 5a (C5/C5a), tissue inhibitors of metalloproteinases-1 (TIMP-1), macrophage colony-stimulating factor (M-CSF) and soluble intercellular adhesion molecule-1 (sICAM-1), respectively. Compared with the normal group, the expression levels of M-CSF, TIMP-1 and C5/C5a in the CIA group were decreased. Following treatment with MCL or MTX, all mice recovered to differing degrees; this was increasingly evident following MTX treatment. The cytokine B-Lymphocyte chemoattractant (BLC) was only present in the MCL group (Fig. 3B). The results of the cytokine analysis and histopathological detection indicated that MCL and MTX were effective in the CIA model. Notably MCL may have a more potent effect than MTX with regard to the effects on autoimmunity.

\section{Discussion}

$\mathrm{RA}$ is one of the most common systemic autoimmune diseases (17). It can result in the progressive destruction of diseased cartilage and joints, severe disability, high social cost and even shortened lifespan (19-21). Previous studies have shown that the CIA model can lead to peripheral arthritis in multiple joints, local joint swelling or even joint deformity. The clinical manifestations, laboratory parameters, immunological and pathological changes are similar to those exhibited in human RA $(1,22)$, therefore, it may be utilized as an animal model of RA. In the present study, DBA/1 mice were used to establish the CIA model and the results obtained suggested that the CIA model in this experiment was successful and stable. The arthritis scores of the MCL group were slightly higher than those of the MTX group, however, the two scores were lower than that of the model group. This suggests that, similarly to MTX, MCL possesses the ability to treat RA. MTX is most commonly used as a disease-modifying antirheumatic drug (DMARD) and has antiproliferative and anti-inflammatory effects (10). In the current study, the histopathological changes of the CIA mice that were treated with MTX were observed to be significantly improved. As the results of the MCL group almost unanimously correspond with the MTX group, this suggests that MCL may also exhibit antiproliferative and anti-inflammatory potential.
The serum cytokines measured in the present study were C5/C5a, TIMP-1, M-CSF, sICAM-1, interferon- $\gamma($ IFN- $\gamma$ ) and BLC. C5/C5a is essential in the innate immune response and evidence now suggests that it may also be involved in adaptive immunity. C5a can bind to the surface of immune cells, such as macrophages, neutrophils and T-cells. It is a well-established receptor that initiates G-protein-coupled signaling via mitogen-activated protein kinase pathways, thereby inducing the synthesis of cytokines such as tumor necrosis factor- $\alpha$ and interleukin- $6(23,24)$. TIMPs are well recognized for their role in extracellular matrix remodeling by controlling the activity of matrix metalloproteinases (MMPs); of these, TIMP-1 with its major target proMMP-9 participate in the regulation of multiple cell functions, including proliferation and migration $(25,26)$. M-CSF is produced by osteoblasts, synovial and periodontal fibroblasts. Combined with the receptor activation of nuclear factor- $\kappa \mathrm{B}$ ligand, it can regulate osteoclastic differentiation and participate in the chronic inflammatory processes that often aggravate bone loss, such as RA (27). The results showed that, following treatment with MCL and MTX, C5/C5a, TIMP-1 and M-CSF recovered to different degrees, particularly the level of TIMP-1 in the MTX group. This suggests that MCL and MTX may modulate the progression of RA via regulation of immunity, cell proliferation, migration and the chronic inflammatory process . sICAM-1 has been shown to have anti-inflammatory effects (28). In the present study, the level of sICAM-1 in the MCL group is lower than that of the MTX and CIA groups, however the sICAM-1 level in the MTX group is similar to that of the CIA group. This indicates that MTX and MCL have extremely limited involvement in the regulation of sICAM-1, particularly MCL. BLC was only present in the MCL group; this phenomenon indicated that MCL may also be involved in autoimmune regulation. IFN- $\gamma$ was only present in the normal DBA/1 mice, which may indicate no association with RA.

Although MTX is a commonly used DMARD for the clinical treatment of RA, there are several disadvantages, including the fact that a significant number of patients require additional treatments in order to control the disease process, either concurrently, or following treatment with MTX. The present study demonstrated that MCL has extremely similar therapeutic effects to MTX, suggesting that MCL may be a potential alternative drug for the treatment of RA. In addition to the existing results, further investigation is required to gain an improved understanding of MCL in the treatment of $\mathrm{RA}$. We propose that MCL may participate in the regulation of the autoimmune system in RA, and therefore suggest that future studies may investigate the underlying mechanisms of its effect on the immune function in RA in greater depth, as well as conducting in vivo studies. Furthermore, studies on the toxicity of MCL are required to demonstrate the clinical applications of this therapy.

\section{Acknowledgements}

This study was supported by grants from the Natural Science Key Program Foundation of Tianjin Technology Commission of China (grant no. 14JCZDJC34900), the National Natural Science General Program Foundation of China 
(grant no. 81170510) and the Major Program Foundation of China (grant no. 81090410).

\section{References}

1. Zhang Y, Xu W, Li H, et al: Therapeutic effects of total alkaloids of Tripterygium wilfordii Hook f. on collagen-induced arthritis in rats. J Ethnopharmacol 145: 699-705, 2013.

2. Vingsbo-Lundberg C, Nordquist N, Olofsson P, et al: Genetic control of arthritis onset,severity and chronicity in a model for rheumatoid arthritis in rats. Nat Genet 20: 401-404, 1998.

3. Choy EH and Panayi GS: Cytokine pathways and joint inflammation in rheumatoid arthritis. N Engl J Med 344: 907-916, 2001.

4. Lee Dm and Weinblatt MB: Rheumatoid arthritis. Lancet 15 903-911, 2001.

5. Schall A and Reiser O: Synthesis of biologically active guaianolides with a trans-annulated lactone moiety. Eur J Org Chem 2008: 2353-2364, 2008.

6. Ma WW, Shi QQ, Ding YH, et al: Synthesis of micheliolide derivatives and their activities against AML progenitor cells. Molecules 18: 5980-5992, 2013.

7. Zhai JD, Li D, Long J, et al: Biomimetic semisynthesis of arglabin from parthenolide. J Org Chem 77: 7103-7107, 2012.

8. Ghantous A, Sinjab A, Herceg Z and Darwiche N: Parthenolide: from plant shoots to cancer roots. Drug Discov Today 18: 894-905, 2013

9. Zhang Q, Lu Y, Ding Y, et al: Guaianolide sesquiterpene lactones, a source to discover agents that selectively inhibit acute myelogenous leukemia stem and progenitor cells. J Med Chem 55: 8757-8769, 2012.

10. Sokka $\mathrm{T}$ and Pincus T: Contemporary disease modifying antirheumatic drugs (DMARD) in patients with recent onset rheumatoid arthritis in a US private practice:methotrexate as the anchor drug in $90 \%$ and new DMARD in $30 \%$ of patients. J Rheumatol 29: 2521-2524, 2002.

11. Swierkot J and Szechiński J: Methotrexate in rheumatoid arthritis. Pharmacol Rep 58: 473-492, 2006.

12. Cronstein BN: Low-dose methotrexate: a mainstay in the treatment of rheumatoid arthritis. Pharmacol Rev 57: $163-172,2005$

13. Pincus T, Yazici Y, Sokka T, et al: Methotrexate as the "anchor drug" for the treatment of early rheumatoid arthritis. Clin Exp Rheumatol 21: S179-S185, 2003.
14. Van der, Heijden JW, Dijkmans BA, et al: Drug Insight: resistance to methotrexate and other disease-modifying antirheumatic drugs--from bench to bedside. Nat Clin Pract Rheumatol 3: 26-34, 2007.

15. Brand DD, Latham KA and Rosloniec EF: Collagen-induced arthritis. Nat Protoc 2: 1269-1275, 2007.

16. Suszko A and Obminska-Mrukowicz B: Influence of polysaccharide fractions isolated from Caitha palustris L. on the cellular immune response in collagen-induced arthritis (CIA) in mice. A comparison with methotrexate. J Ethnopharmacol 145: 109-117, 2013.

17. Cuzzocrea S, Ayroldi E, Di Paola R, et al: Role of glucocorticoid-induced TNF receptor family gene (GITR) in collagen-induced arthritis. FASEB J 19: 1253-1265, 2005.

18. Xinqiang S, Fei L, Nan L, Yuan L, et al: Therapeutic efficacy of experimental rheumatoid arthritis with low-dose methotrexate by increasing partially CD4+CD25+Treg cells and inducing Th1 to Th2 shift in both cells and cytokines. Biomed Pharmacother 64: 463-471, 2010.

19. Firestein GS: Evolving concepts of rheumatoid arthritis. Nature 423: 356-361, 2003.

20. Wildbaum G, Nahir MA and Karin N: Beneficial autoimmunity to proinflammatory mediators restrains the consequences of self-destructive immunity. Immunity 19: 679-688, 2003.

21. Sinha AA, Lopez MT and McDevitt HO: Autoimmune diseases: the failure of self tolerance. Science 248: 1380-1388, 1990.

22. Zhou J, Xiao C, Zhao L, et al: The effect of triptolide on CD4+ and CD8+ cells in Peyer's patch of SD rats with collagen induced arthritis. Int Immunopharmacol 6: 198-203, 2006.

23. Ma N, Xing C, Xiao H, et al: C5a regulates IL-12 (+) DC migration to induce pathogenic Th1 and Th17 cells in sepsis. PLoS One 8: e69779, 2013.

24. Laudes IJ, Chu JC, Huber-Lang M, et al: Expression and function of C5a receptor in mouse microvascular endothelial cells J Immunol 169: 5962-5970, 2002.

25. Ries C: Cytokine functions of TIMP-1. Cell Mol Life Sci 71: 659-672, 2014.

26. Djafarzadeh R, Mojaat A, Vicente AB, et al: Exogenously added GPI-anchored tissue inhibitor of matrix metalloproteinase-1 (TIMP-1) displays enhanced and novel biological activities. Biol Chem 385: 655-663, 2004.

27. Souza PP and Lerner UH: The role of cytokines in inflammatory bone loss. Immunol Invest 42: 555-622, 2013.

28. Fei X, Hongxiang Z, Qi C, Daozhen C: Maternal plasma levels of endothelial dysfunction mediators including AM, CGRP, sICAM-1 and tHcy in pre-eclampsia. Adv Clin Exp Med 21: 573-579, 2012. 